\title{
MiR-291b-3p Induces Apoptosis in Liver Cell Line NCTC1469 by Reducing the Level of RNA-binding Protein HuR
}

\author{
Jun Guo ${ }^{a, b} \quad$ Meng Lia ${ }^{a, b} \quad$ Xiangyu Meng ${ }^{a, b}$ JingZhe Sui ${ }^{b} \quad$ Lin Dou $^{b}$ Weiqing Tang ${ }^{b}$ \\ Xiuqing Huang ${ }^{b}$ Yong Man ${ }^{b}$ Shu Wang ${ }^{b}$ Jian Lia,b \\ aPeking University Fifth School of Clinical Medicine, Beijing Hospital, ${ }^{\mathrm{b}}$ The Key Laboratory of Geriatrics, \\ Beijing Hospital \& Beijing Institute of Geriatrics, Ministry of Health, Beijing, China
}

\section{Key Words}

miR-291b-3p • NCTC1469 cell • Apoptosis • HuR, Bcl-2

\begin{abstract}
Background: There is increasing evidence that miRNAs are involved in cellular apoptosis. However, the specific role of miR-291b-3p in apoptosis has not been elucidated. In the present study, we investigated the effect of miR-291b-3p on NCTC1469 cell growth and apoptosis. Methods: Cell viability and apoptosis were examined in NCTC1469 cells transfected with miR-291b-3p mimics, inhibitor miRNA or negative control. Using computational miRNA target prediction databases, HuR was predicted as a target of miR-291b-3p. Luciferase assay, immunofluorescence and western blot were used to further explore the effects of miR-291b-3p on HuR expression. In addition, the effect of HuR on cell apoptosis was evaluated using a HuRspecific siRNA. Results: TNF- $\alpha$-induced hepatocyte apoptosis was accompanied by enhanced expression of miR-291b-3p, suggesting that miR-291b-3p might contribute to the apoptotic process. Follow-up experiments showed that upregulation of miR-291b-3p decreased cell viability and induced NCTC1469 cell apoptosis. Additionally, similar to the activity of miR-519, which is another member of the same miRNA family, miR-291b-3p suppressed HuR translation through binding to the HuR coding region (CR). We further showed that the downregulation of HuR expression by miR-291b-3p was accompanied by reduced $\mathrm{Bcl}-2$ expression. Moreover, knockdown of HuR also impaired $\mathrm{Bcl}-2$ expression and increased the ratio of $\mathrm{Bax} / \mathrm{Bcl}-2$. More significantly, downregulation of miR-291b-3p failed to increase Bcl2 expression in NCTC1469 cells that were co-transfected with siRNA-HuR. Finally, inhibition of miR-291b-3p led to reduced apoptosis, while knockdown of HuR by siRNA promoted apoptosis, even in NCTC1469 cells that were co-transfected with the miR-291b-3p inhibitor. Conclusion: The current data suggested that miR-291b-3p contributed to NCTC1469 cell apoptosis by regulating the expression of HuR, which in turn increased Bcl-2 stability.
\end{abstract}

Copyright $($ C 2014 S. Karger AG, Basel 


\section{Cellular Physiology and Biochemistry}

Cell Physiol Biochem 2014;33:810-822

\begin{tabular}{l|l}
\hline DOI: $10.1159 / 000358654$ & (C) 2014 S. Karger AG, Basel
\end{tabular}

www.karger.com/cpb

Guo et al.: MiR-291b-3p Induces Cell Apoptosis

\section{Introduction}

MiRNAs are small non-coding RNAs that potently regulate gene expression in mammalian cells. The primary transcript of miRNA is initially transcribed by RNA polymerase II or RNA polymerase III. In the nucleus, the primary transcript is processed by Drosha and transformed into the pri-miRNA with approximately 60 to 110 nucleotides. The transcript is then transported to the cytoplasm and further cleaved to the mature form by Dicer. The mature miRNA is incorporated into the RNA-induced silencing complex (RISC) [1]. The complex is tethered to a complementary sequence within the 3'-untranslated region (UTR) of the target mRNA, resulting in the suppression of gene translation and expression. It has been suggested that over $30 \%$ of all human genes are regulated by miRNAs, many of which are involved in important cellular pathways [2]. In recent years, numerous investigations have implicated miRNA dysregulation during cellular apoptosis, leading to additional studies on the involvement of miRNAs and their target genes in apoptotic pathways. For instance, miR-150 and miR-630 were shown to induce pancreatic cancer cell apoptosis by targeting IGF-1R, and miR-21 was shown to have anti-apoptotic functions by targeting pro-apoptotic genes, such as FasL, PTEN and PDCD4 [3, 4].

In addition to miRNAs, AU-rich elements (AREs)-mediated transcript degradation is considered as another important gene regulation mechanism at the post-transcriptional level [5]. Undoubtedly, AREs are important cis-elements that control cell growth and proliferation because a number of cancer-related transcripts contain AREs in the 5'-untranslated region (UTR) or 3'-UTR, such as cytokines, growth factors and invasion factors [6]. AREs are recognized by RNA binding proteins, which then affect gene expression by changing the stability and translation efficiency of mRNAs [6]. The human embryonic lethal abnormal vision-like (ELAV) protein HuR is an RNA binding protein in the Hu family [7]. It is widely expressed in mammalian cells and can bind to AREs to adjust mRNA stability.

According to the number and distribution of AUUUA nucleotides, AREs can be divided into three types [8]. Type I contains several copies of the AUUUA motif; type II has at least two copies of UUAUUUA (U/A) (U/A); and type III lacks the typical AUUUA motif in the U-rich regions. HuR can recognize and bind to all three types of AREs in the 5'-UTR or 3'-UTR of mRNAs. For most of these mRNAs, HuR was reported to increase their stability [9-11]. It has also been shown that, while HuR is predominantly localized in the nucleus, it can be shuttled between the nucleus and the cytoplasm. This translocation allows HuR to efficiently stabilize its target mRNAs [12]. Over the past decades, there has been increasing evidence suggesting that HuR plays a key role in carcinogenesis and cancer progression by regulating the expression of numerous target genes, such as p53, p21, p27, and Bcl-2 [13-15].

Recently, it was reported that many miRNAs, such as miR-9, miR-34a, miR-16, miR-125a, miR-29a, miR-200c and miR-519, can regulate the protein expression of HuR by binding to specific regions in the 3'UTR or 5'UTR of HuR [16-21]. The present study describes a novel miRNA, miR-291b-3p, that participates in HuR regulation. Furthermore, our study shows that inhibition of HuR expression by miR-291b-3p in NCTC1469 cells may induce apoptosis through downregulation of Bcl-2.

\section{Materials and Methods}

\section{Cell culture}

Murine liver cell line NCTC1469 was cultured in Dulbecco's Modified Eagle's Medium (DMEM) supplemented with $10 \%(\mathrm{v} / \mathrm{v}$ ) Horse serum (Atlanta Biolabs), $100 \mathrm{units} / \mathrm{ml}$ penicillin, and $100 \mu \mathrm{g} / \mathrm{ml}$ streptomycin (Life Technologies, Inc.), at $37^{\circ} \mathrm{C}$ in a humidified atmosphere with $5 \% \mathrm{CO}_{2}$.

Isolation of mouse primary hepatocytes

Male C57BL/6J mice (12 weeks old) were provided by the Peking University Health Science Center (Beijing, China). Mouse primary hepatocytes were isolated as previously described [22]. 


\section{Cellular Physiology \\ and Biochemistry}

Cell Physiol Biochem 2014;33:810-822

\begin{tabular}{l|l}
\hline DOI: $10.1159 / 000358654$ & (c) 2014 S. Karger AG, Basel
\end{tabular}

www.karger.com/cpb

Guo et al.: MiR-291b-3p Induces Cell Apoptosis

Transient transfection

Before transfection, $1 \times 10^{5}$ cells per well were seeded in a 6-well plate with $2 \mathrm{ml}$ DMEM culture medium containing serum and antibiotics. Meanwhile, miR-291b-3p mimic, inhibitor, or negative control (Genepharma) was pre-incubated with HiPerFect transfection reagent (QIAGEN) at room temperature for $10 \mathrm{~min}$. The complex was then transfected into the NCTC1469 cells at a final concentration of $50 \mathrm{nM}$. The transfected cells were incubated under normal growth conditions for another $48 \mathrm{~h}$.

\section{RNA extraction and real-time PCR}

Total RNA was extracted from NCTC1469 cells with Trizol (Invitrogen) according to the manufacturer's instructions. The quality of the RNA samples was monitored by analysis of $\mathrm{OD}_{260} / \mathrm{OD}_{280}$.

To measure the level of miR-291b-3p, a quantitative 2-step RT-PCR assay was performed using SYBR Green Supermix (Bio-Rad) in a BIO-RAD iCycler iQ real-time PCR detection system.

For the quantitation of miRNA, $1 \mu \mathrm{g}$ of the total RNA were reverse transcribed using Taq-Man MicroRNA Reverse Transcription Kit (Applied Biosystems) with specific primers for miR-291b-3p and U6 (Shanghai Sangon Technology), and subsequently the PCR amplifications were performed in $20 \mu$ reaction volumes

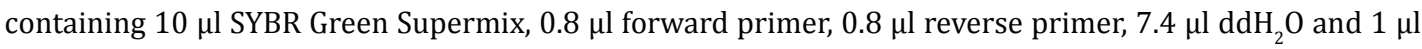
template cDNA. The thermal cycling conditions were a hot start step at $95^{\circ} \mathrm{C}$ for $10 \mathrm{~min}$, followed by 40 cycles at $95^{\circ} \mathrm{C}$ for $15 \mathrm{~s}$ and $60^{\circ} \mathrm{C}$ for $1 \mathrm{~min}$. The relative level of miR-291b-3p was determined using the $2^{-\Delta \Delta \mathrm{Ct}}$ analysis method, where U6 was used as the endogenous control. Nucleotide primers used for reverse transcription were as follows (5'-3'): miR-291b-3p, GTCGTATCCAGTGCAGGGTCCGAGGTATTCGCACTGGATACGACACAAAC; U6, GTCGTATCCAGTGCAGGGTCCGAGGTATTCGCACTGGATACGACAAATATG.

The primers used for real-time PCR were as follows (5'-3'): miR-291b-3p forward, GCAAAGTGCATCCATTTTGTTTGT; U6 forward, GCGCGTCGTGAAGCGTTC; Universal reverse primer, GTGCAGGGTCCGAGGT.

\section{Protein extraction and western blot}

Proteins were extracted from NCTC1469 cells with RIPA buffer (1\% TritonX-100, 150 mmol/L NaCl, 5 $\mathrm{mmol} / \mathrm{L}$ EDTA, and $10 \mathrm{mmol} / \mathrm{L}$ Tris-HCl (pH 7.0)) (Solarbio, China) supplemented with a protease inhibitor cocktail (Sigma). The cell lysates were separated by 10\% SDS-PAGE and transferred electrophoretically onto PVDF membranes. After blocking with 8\% milk in PBS (pH 7.5), the membranes were incubated with the following specific primary antibodies overnight: anti-HuR, anti-bcl-2, anti-bax and anti- $\beta$-actin (SantaCruz Biotechnology, Inc.). The appropriate HRP-conjugated anti-rabbit IgG secondary antibody (Abmart, all at 1:5000) was subsequently applied, and immunodetection was performed using the ECL Plus detection system (Millipore) according to the manufacturer's instructions. $\beta$-actin was used as the internal control.

\section{Dimethyl thiazolyl diphenyl tetrazolium (MTT) assay}

To explore the effect of miR-291b-3p on cell viability, 5,000 cells per well in a $100 \mu \mathrm{l}$ medium were seeded in 96-well plates and transfected with miR-291b-3p mimics (50 nM) or negative control-miRNA mimics (50 nM), as described above. Every $24 \mathrm{~h}$ after transfection, $20 \mu \mathrm{l}$ of the 3-(4,5-dimethylthiazol-2-yl)2,5-diphenyltetrazolium bromide (MTT) reagent (Solarbio, China) was added to wells and were incubated with the cells for $4 \mathrm{~h}$. After removing the medium, the blue formazan was dissolved with $200 \mu \mathrm{l}$ dimethyl sulfoxide (DMSO) and the absorbance was measured at $550 \mathrm{~nm}$. Wells containing only NCTC1469 cells served as blanks.

\section{Hoechst 33258 staining}

NCTC1469 cells were cultured in 6-well plates. After 48 h transfection with miR-291b-3p mimics, inhibitors or negative control, cells were washed with PBS and stained with Hoechst $33258(10 \mu \mathrm{g} / \mathrm{ml})$ for 5 min before being washed three times with PBS.

\section{Immunofluorescence}

NCTC1469 cells were cultured on 6-well chamber slides and fixed with 4\% paraformaldehyde for 10 $\min$ at $-20^{\circ} \mathrm{C}$. The slides were washed in PBS for three times, and incubated with a polyclonal antibody against HuR (1:50 diluted in PBS with 1\% BSA, 50 $\mu \mathrm{l} / \mathrm{slide}$ ) for $2 \mathrm{~h}$ at room temperature. After washing with PBS for three times ( 5 min per time), the slides were incubated with TRITC-conjugated anti-mouse 


\section{Cellular Physiology and Biochemistry}

Cell Physiol Biochem 2014;33:810-822

\begin{tabular}{l|l}
\hline DOI: $10.1159 / 000358654$ & (C) 2014 S. Karger AG, Basel
\end{tabular}

Guo et al.: MiR-291b-3p Induces Cell Apoptosis

IgG (1:100 diluted in PBS with 1\% BSA, 50 $\mathrm{l} /$ slide) for $1 \mathrm{~h}$ at room temperature. Three times after washing the slides in PBS, the slides were incubated with Hoechst $33258(10 \mu \mathrm{g} / \mathrm{ml})$ for $5 \mathrm{~min}$. Then, the slides were washed again and examined using a fluorescence microscope.

Quantification of apoptotic cells

To quantify apoptotic cells, flow cytometry was performed with an Annexin V-fluorescein-5isothiocyanate apoptosis detection kit (Bio-vision, USA). $48 \mathrm{~h}$ after transfection with miR-291b-3p mimics $(50 \mathrm{nM})$ and a negative control (50 nM), NCTC1469 cells were harvested in a $5 \mathrm{ml}$ tube. Then, the cells were washed with cold PBS and resuspended at a final concentration of $1 \times 10^{6}$ cells $/ \mathrm{ml}$. FITC-AnnexinV (5 ul) and propidium iodide were gently mixed and incubated with the cells for $15 \mathrm{~min}$ at a room temperature. After incubation, the samples were analyzed by flow cytometry within $1 \mathrm{~h}$.

\section{Luciferase target assay}

For luciferase assay, the 3' untranslated region (UTR) or coding region of HuR including the binding sites for miR-291b-3p was amplified from NCTC1469 cells by using the following primers (restriction sites are underlined):

HuR-CD-N-XhoI 5' CCCTCGAG CTGGCTCTGGAATCATTGCT

HuR -CD-C-XhoI 5' CCCTCGAG AGGCAGTCTTCGGTTCTTGA

HuR-UTR-N-XhoI 5' CCCTCGAGCCTATATGGGGTTGCTTCCA

HuR-UTR-C-Xhol 5' CCCTCGAG CCAACCAGCCTTCTTTTCTG

PCR was performed with genome DNA isolated from NCTC1469 cells and the PCR product was then digested with Xhol (NEB). Then, the fragment was inserted into the Xhol-linearlized pGL3 (Promega) luciferase reporter vector. To ensure the ligation efficiency, both the PCR products and the XhoI-linearlized pGL3 vectors were treated with thermosensitive alkaline phosphatese (NEB) at $80{ }^{\circ} \mathrm{C}$ for $20 \mathrm{~min}$. Details of PCR procedures are described as follows: a hot start step at $95^{\circ} \mathrm{C}$ for $10 \mathrm{~min}$, followed by 40 cycles at $95^{\circ} \mathrm{C}$ for $15 \mathrm{~s}$ and $55^{\circ} \mathrm{C}$ for $45 \mathrm{~s}, 72^{\circ} \mathrm{C}$ for $30 \mathrm{~s}$.

To conduct luciferase reporter assay, 5000 cells per well in a $100 \mu \mathrm{l}$ medium were seeded in 96 -well plates. After incubation overnight, the cells were transfected with the modified firefly luciferase vector $(500 \mathrm{ng} / \mu \mathrm{l})$ with lipofectamine TM 2000 Reagent (Invitrogen) according to the manufacturer's instruction. $48 \mathrm{~h}$ after transfection, the firefly and renilla luciferase activities were measured with the Dual-luciferase reporter assay system (Promega). To control the transfection efficiency, Firefly activity was normalized to renilla activity.

\section{Inhibition of HuR by RNA interference}

HuR-specific siRNA (siHuR) and negative control were purchased from Genepharma. $1 \times 10^{5}$ cells per well in a 6-well plate were transfected with $50 \mathrm{nM}$ siHuR or negative control for $48 \mathrm{~h}$ using HiperFect transfection reagent (QIAGEN) as described above.

Statistical analysis

Data were presented as mean \pm SE from 3 independent experiments. Statistical analysis was carried out with Student's $t$ test. $P<0.05$ was considered as statistically significant difference.

\section{Results}

TNF- $\alpha$ induced hepatocyte apoptosis, accompanied by enhanced level of miR-291b-3p

In previous study, we analyzed miRNAs in the livers of $\mathrm{db} / \mathrm{db}$ mice $(n=5)$ and control mice $(n=5)$ by miRNA microarray and verified by real-time PCR. The results showed that increased level of miR-291b-3p in the livers was accompanied by enhanced TNF- $\alpha$ level in the serum of $\mathrm{db} / \mathrm{db}$ mice (data not shown). In the present study, to identify the impact of miR-291b-3p on cell apoptosis induced by TNF- $\alpha$, we extended the observation in the $\mathrm{db} /$ $\mathrm{db}$ mice to murine liver cell line NCTC1469. To investigate the effect of TNF- $\alpha$ on hepatocyte apoptosis, murine liver cell line NCTC1469 was treated with $20 \mathrm{ng} / \mathrm{ml}$ TNF- $\alpha$ for $48 \mathrm{~h}$. Hoechst staining showed that TNF- $\alpha$ significantly induced apoptosis in these cells (Fig 1. A). 
A

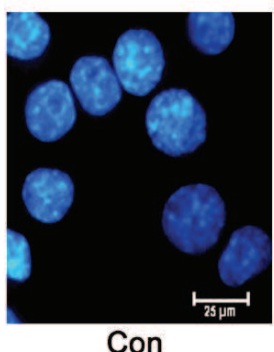

B

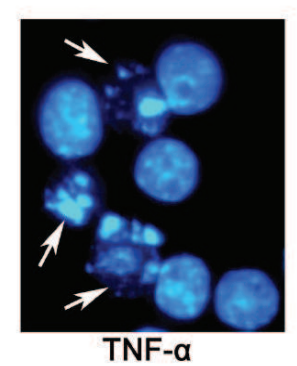

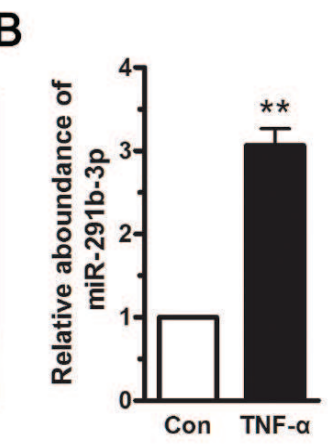

C

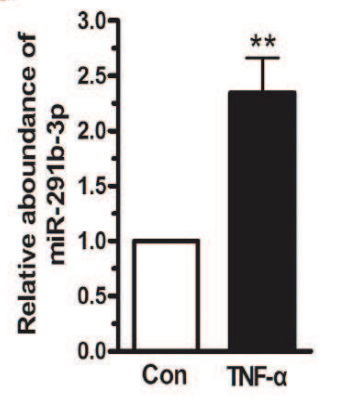

Fig. 1. TNF- $\alpha$ induced hepatocyte apoptosis, accompanied by enhanced level of miR-291b-3p. Murine liver cell line NCTC1469 was treated with $20 \mathrm{ng} / \mathrm{ml} \mathrm{TNF-} \alpha$ for $48 \mathrm{~h}$. Hoechst staining showed that TNF- $\alpha$ significantly induced NCTC1469 cell apoptosis (A). White arrow indicated apoptotic cells. The level of miR291b-3p was increased in the NCTC1469 cells (B) and the primary hepatic cells (C) treated with TNF- $\alpha$ compared with the negative control. Data represent the means \pm SEM, $n=3$ independent experiments. ${ }^{* *} p<0.01$ versus control.

Fig. 2. MiR-291b-3p regulated NCTC1469 cell viability. NCTC1469 cells were transfected with miR-291 mimics, inhibitor or negative control for 24, 48, $72 \mathrm{~h}$. Upregulation of miR-291b-3p decreased cell viability by $25 \%$ and $30 \%$ at 48 $\mathrm{h}$ and $72 \mathrm{~h}(\mathrm{~A})$, while

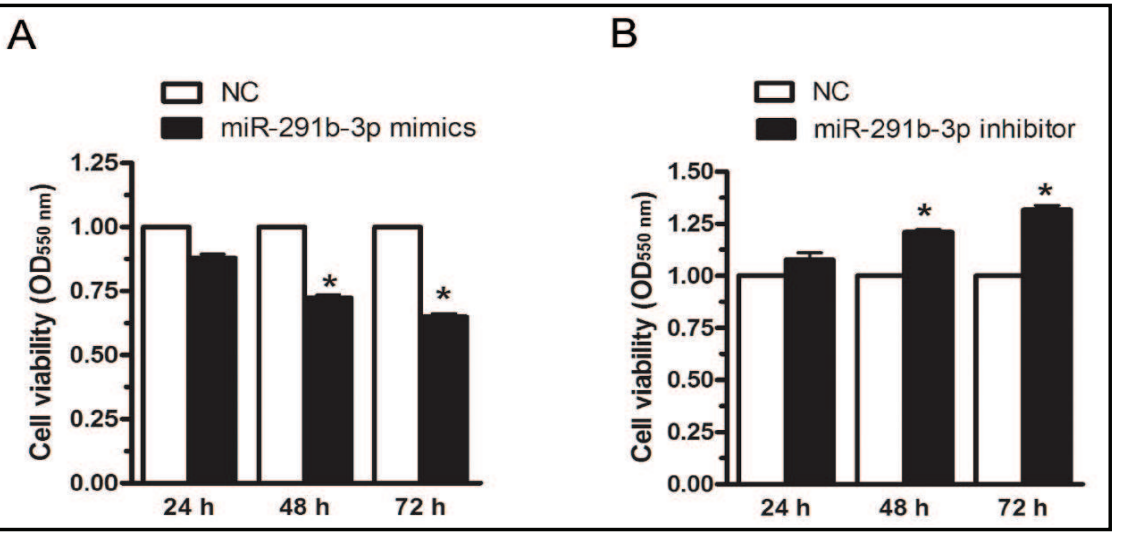
downregulation of miR-291b-3p enhanced cell viability in the NCTC1469 cells by $20 \%$ and $30 \%$ at $48 \mathrm{~h}$ and $72 \mathrm{~h}$, respectively (B), as analyzed by MTT assay. Data represent the means \pm SEM, $n=6$ independent experiments. ${ }^{*} p<0.05$ versus control.

Moreover, we also explored the effect of TNF- $\alpha$ on the expression of miR-291b-3p in both NCTC1469 cells and primary hepatic cells. Our data indicated that the TNF- $\alpha$ treatment significantly increased the level of miR-291b-3p in both cell populations (Fig 1. B and C). These in vitro observations indicated that miR-291b-3p might contribute to TNF- $\alpha$-induced apoptosis of hepatocytes.

\section{MiR-291b-3p regulated NCTC1469 cell viability}

To determine the effect of miR-291b-3p on cell viability, NCTC1469 cells were transfected with miR-291 mimics, inhibitor or negative control for 24, 48, and $72 \mathrm{~h}$. As shown in Fig 2. A, upregulation of miR-291b-3p by the mimics decreased cell viability by $25 \%$ and $30 \%$ at $48 \mathrm{~h}$ and $72 \mathrm{~h}$, respectively, while downregulation of miR-291b-3p by the inhibitor enhanced cell viability by $20 \%$ and $30 \%$ at $48 \mathrm{~h}$ and $72 \mathrm{~h}$, respectively (Fig 2 . B). These results demonstrated that miR-291b-3p could modulate NCTC1469 cell viability.

\section{Upregulation of miR-291b-3p induced NCTC1469 cell apoptosis}

We next observed that upregulation of miR-291b-3p enhanced cell apoptosis by nearly fourfold versus the negative control in NCTC1469 cells (Fig 3. A). Hoechst 33342 staining 


\section{Cellular Physiology and Biochemistry}

Cell Physiol Biochem 2014;33:810-822

Fig. 3. Upregulation of miR-291b-3p induced NCTC1469 cell apoptosis. MiR-291b-3p mimics or inhibitor were transfected into NCTC1469 cells for $48 \mathrm{~h}$. Upregulation of miR-291b-3p enhanced cell apoptosis by $130 \%$ versus negative control in the NCTC1469 cells (A), as tested by Annexin V and PI kit. (B) The apoptotic cells increased in the NCTC1469 cells transfected with miR291b-3p mimics, as examined by Hoechst 33342 staining. White arrow indicated apoptotic cells. As assessed by Western blot, upregulation of miR-291 could decrease the level of $\mathrm{Bcl} 2$ and increase the levels of Bax and Bim (C), while suppression of miR-291b$3 p$ could elevate the expression level of Bcl-2, and reduced the levels of Bax and Bim (D). Data represent the means $\pm \mathrm{SEM}, \mathrm{n}=3$ independent experiments. ${ }^{*} p<0.05$, ** $p<0.01$ versus control.

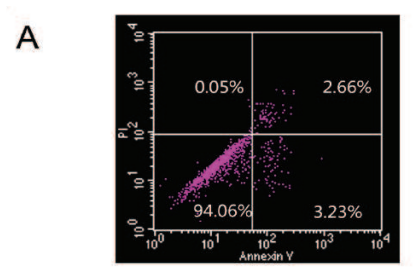

NC

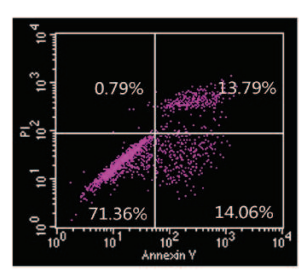

miR-291b-3p mimics

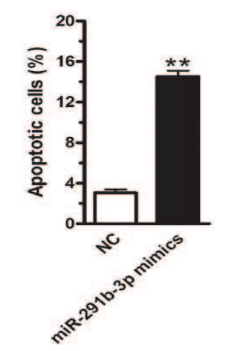

B

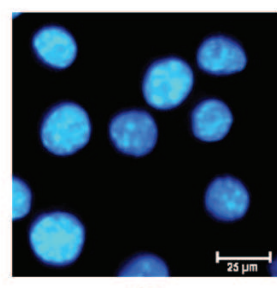

NC

C

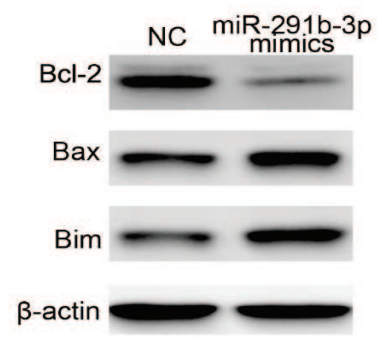

D

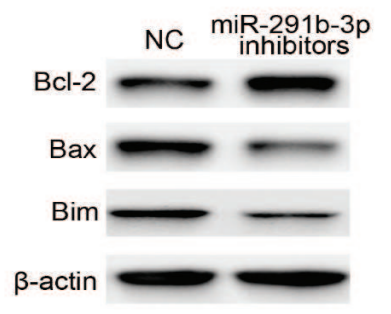

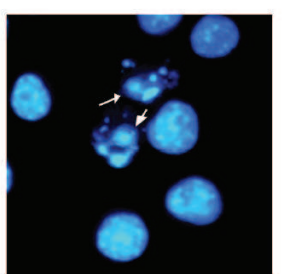

miR-291b-3p mimics
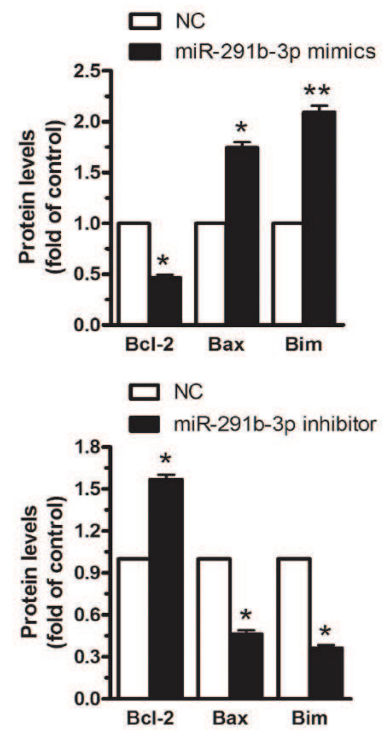

also verified that the number of apoptotic cells were higher in the NCTC1469 cells that were transfected with miR-291b-3p mimics when compared with the negative control (Fig 3. B). Together these data indicated that upregulation of miR-291b-3p induced NCTC1469 cell apoptosis. Furthermore, western blot analysis of apoptotic protein expression showed that upregulation of miR-291 resulted in lower expression of Bcl2 and higher expression of Bax and Bim (Fig 3. C). Moreover, suppression of miR-291b-3p with the specific inhibitor restored the expression of Bcl-2 and reduced the levels of Bax and Bim (Fig 3. D). Taken together, these results indicated that miR-291b-3p might induce NCTC1469 cell apoptosis partially through the regulation of Bcl-2, Bax and Bim expression.

MiR-291b-3p directly bound to the coding region of HuR

MiR-291b-3p belongs to the same family of miRNAs that includes miR-519b-3p, miR519c-3p, and miR-519a. In previous studies, it has been shown that miR-519 reduces cell proliferation by directly regulating HuR expression $[21,23]$. Thus, the sequences of HuR from Mus musculus and Homo sapiens were aligned. As shown in Fig 4. A, the nucleotides in the coding region (CR) of HuR from $902 \mathrm{bp}$ to $923 \mathrm{bp}$ in Mus musculus and from $886 \mathrm{bp}$ to 907 


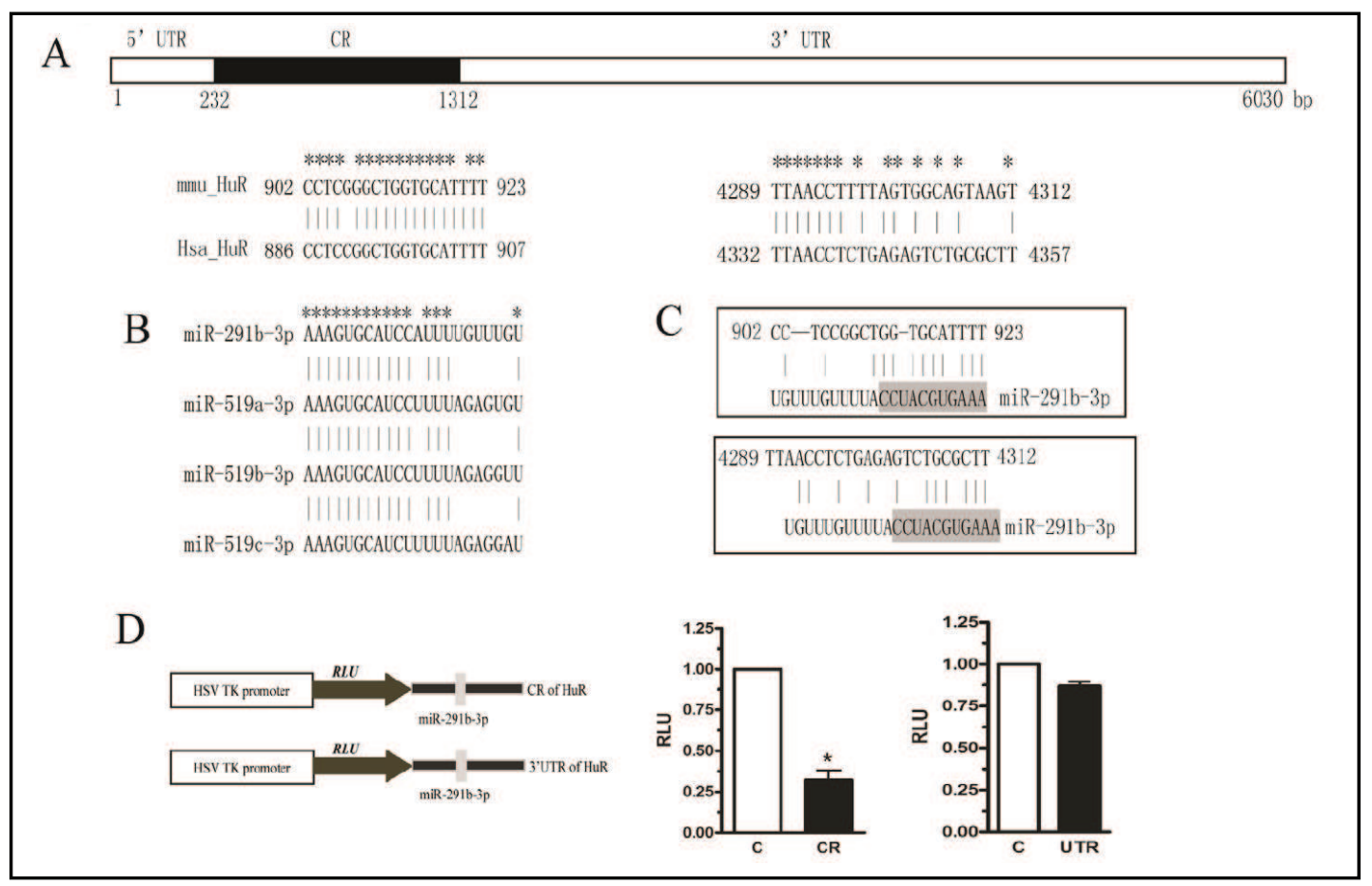

Fig. 4. MiR-291b-3p directly bound to the coding region of HuR. The sequences of HuR from Mus musculus and Homo sapiens were aligned. The nucleotides in the coding region (CR) of HuR from 902 bp to 923 bp in Mus musculus were high conserved with the nucleotides from $886 \mathrm{bp}$ to $907 \mathrm{bp}$ in Homo sapiens. Poor conserved amino acids of HuR in the 3'-untranslated region were defined in Mus musculus and Homo sapiens. The sequence of miR-291b-3p with miR-519b-3p, miR-519c-3p, and miR-519a was also aligned (A). The sequence of miR-291b-3p was highly consistent with miR-519b-3p, miR-519c-3p, and miR-519a (B). MiR-291b-3p from mouse was aligned with the murine HuR mRNA (C). As confirmed by luciferase reporter assay, overexpression of miR-291b-3p could significantly reduce the luciferase activity in the HEK 293T cells transfected with luciferase reporter vector with the HuR-CR region, while the luciferase activity was slightly reduced when HEK cells were co-transfected with luciferase reporter vector containing the HuR-3'-UTR and miR-291b-3p mimics (D). Data represent the means \pm SEM, $n=3$ independent experiments. * $p<0.05$ versus control.

bp in Homo sapiens were highly conserved, and this sequence was confirmed as the target site of miR-519. Poorly conserved amino acids in the 3'-untranslated region of HuR were also defined in Mus musculus and Homo sapiens. Furthermore, we aligned the sequence of miR-291b-3p with those of miR-519b-3p, miR-519c-3p, and miR-519a. Fig. 4B shows that there is high similarity between the four miRNA sequences. Based on the sequence analysis above, murine miR-291b-3p was then aligned with the murine HuR mRNA. The result indicated that HuR may be a potential target gene of miR-291b-3p (Fig 4. C). A luciferase reporter assay was then used to assess whether miR-291b-3p can directly bind to the HuR mRNA. Overexpression of miR-291b-3p significantly reduced the luciferase activity in HEK 293T cells that were transfected with the luciferase reporter vector containing the HuR-CR region. However, the luciferase activity was only slightly reduced when HEK cells were cotransfected with both the luciferase reporter vector containing the HuR-3'-UTR and the miR291b-3p mimics (Fig 4. D). These data suggest that miR-291b-3p directly modulates HuR expression by binding to the coding region of HuR.

Additional validation of HuR modulation by miR-291b-3p

To further confirm that HuR is negatively regulated by miR-291b-3p, HuR expression was analyzed by immunofluorescence. We found that HuR was predominantly located in 


\section{Cellular Physiology \\ and Biochemistry}

Cell Physiol Biochem 2014;33:810-822

Fig. 5. HuR is the host gene of miR-291b-3p. Immunofluorescence revealed that HuR was predominately located in the nucleus and significantly reduced in the NCTC1469 cells transfected with miR291b-3p mimics for $48 \mathrm{~h}(\mathrm{~A})$. As analyzed by Western blot, overexpression of miR$291 b-3 p$ reduced the protein level of $\mathrm{HuR}$ (B), while suppression of miR-291b-3p enhanced HuR level (C). Data represent the means \pm SEM, $\mathrm{n}=3$ independent experiments. $* p<0.05$, ** $p<0.01$ versus control.

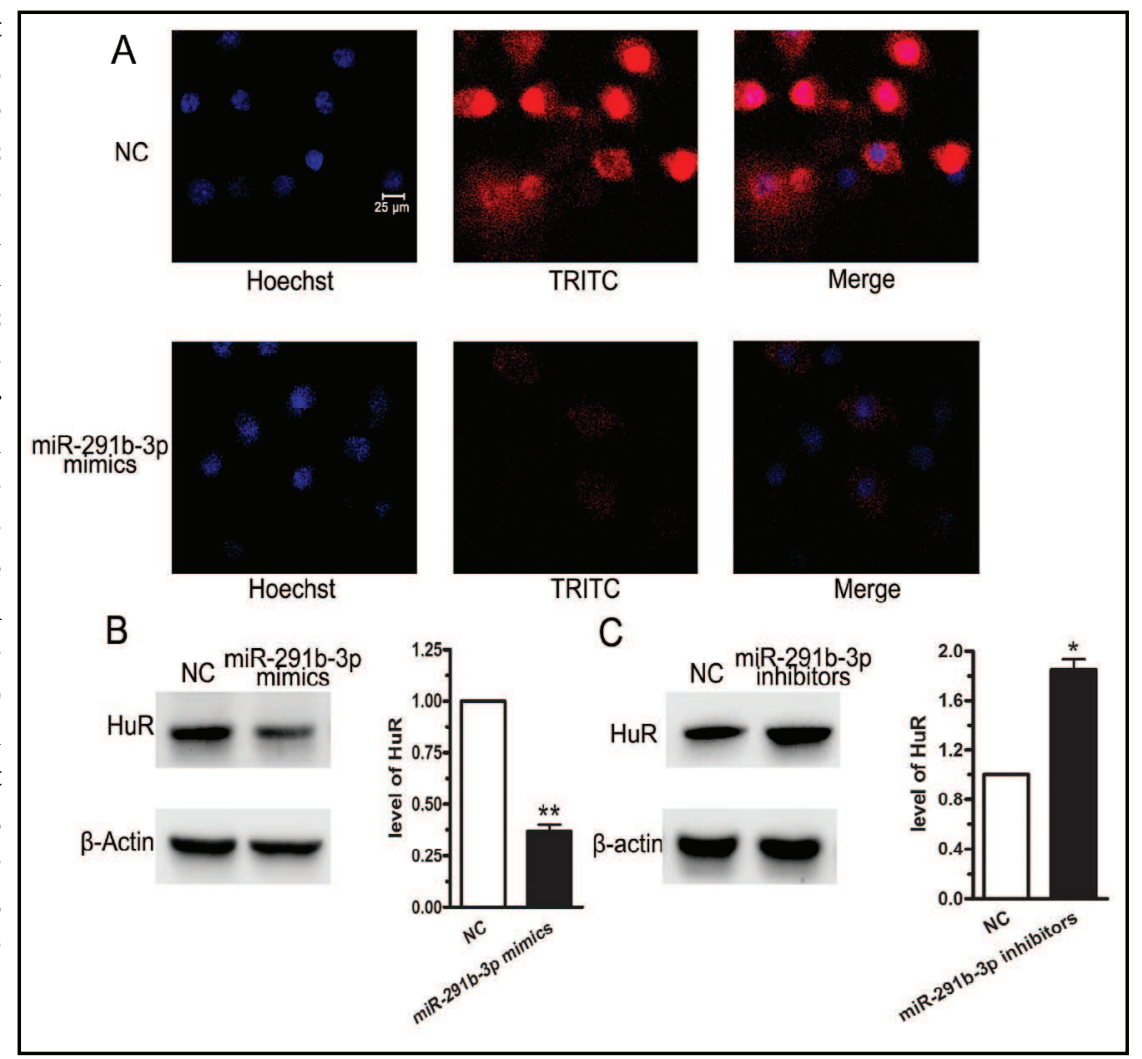

Fig. 6. Suppression of HuR expression could induce apoptosis of NCTC1469 cells. The levels of HuR and Bcl-2 were reduced in the NCTC1469 cells transfected with siRNA of HuR for $48 \mathrm{~h}$, while the Bax expression was not changed (A). When HuR expression was downregulated, cell apoptosis was increased by $123 \%$ in comparison with the control group, as analyzed by flow cytometry (B). Data represent the means \pm SEM, $n=3$ independent experiments. ${ }^{*} p<0.05$, ** $p<0.01$ versus control.
A
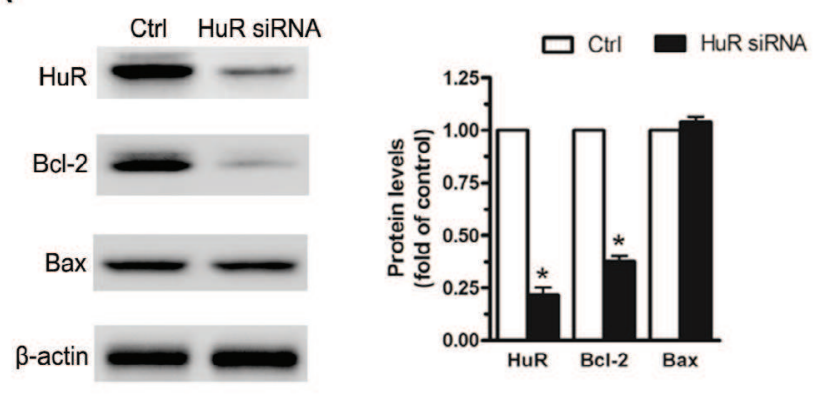

B
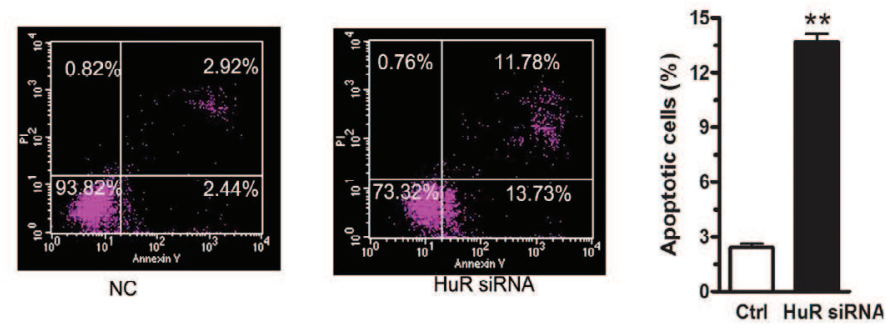

the nucleus $48 \mathrm{~h}$ after the transfection of miR-291b-3p mimics, and the level of HuR was significantly reduced compared with the negative control (Fig 5. A). Furthermore, western blot analysis of HuR expression in cells that were transfected with the miR-291b-3p mimics or inhibitor for $48 \mathrm{~h}$ revealed that overexpression of miR-291b-3p reduced the protein level of HuR (Fig 5. B), while suppression of miR-291b-3p enhanced the level of HuR (Fig 5. C). However, no changes were detected in the mRNA level of HuR under both conditions, indicating that miR-291b-3p affected HuR expression at the post-transcriptional level 


\section{Cellular Physiology and Biochemistry}

Cell Physiol Biochem 2014;33:810-822

Fig. 7. MiR291b-3p induced cell apoptosis by reducing HuR expression. Western blot showed the levels of $\mathrm{HuR}$ and $\mathrm{Bcl} 2$ were reduced in the NCTC1469 cells transfected with miR-291b$3 p$ mimics and siRNA-HuR (A). Downregulation of miR-291b-3p elevated the expression of HuR and $\mathrm{Bcl} 2$, while downregulation of HuR reduced Bcl-2 expression. More significantly, downregulation of miR-291b-3p could no longer increase the level of Bcl2 in the NCTC1469 cells transfected with siRNA-HuR (B). Downregulation of HuR did not affect the level

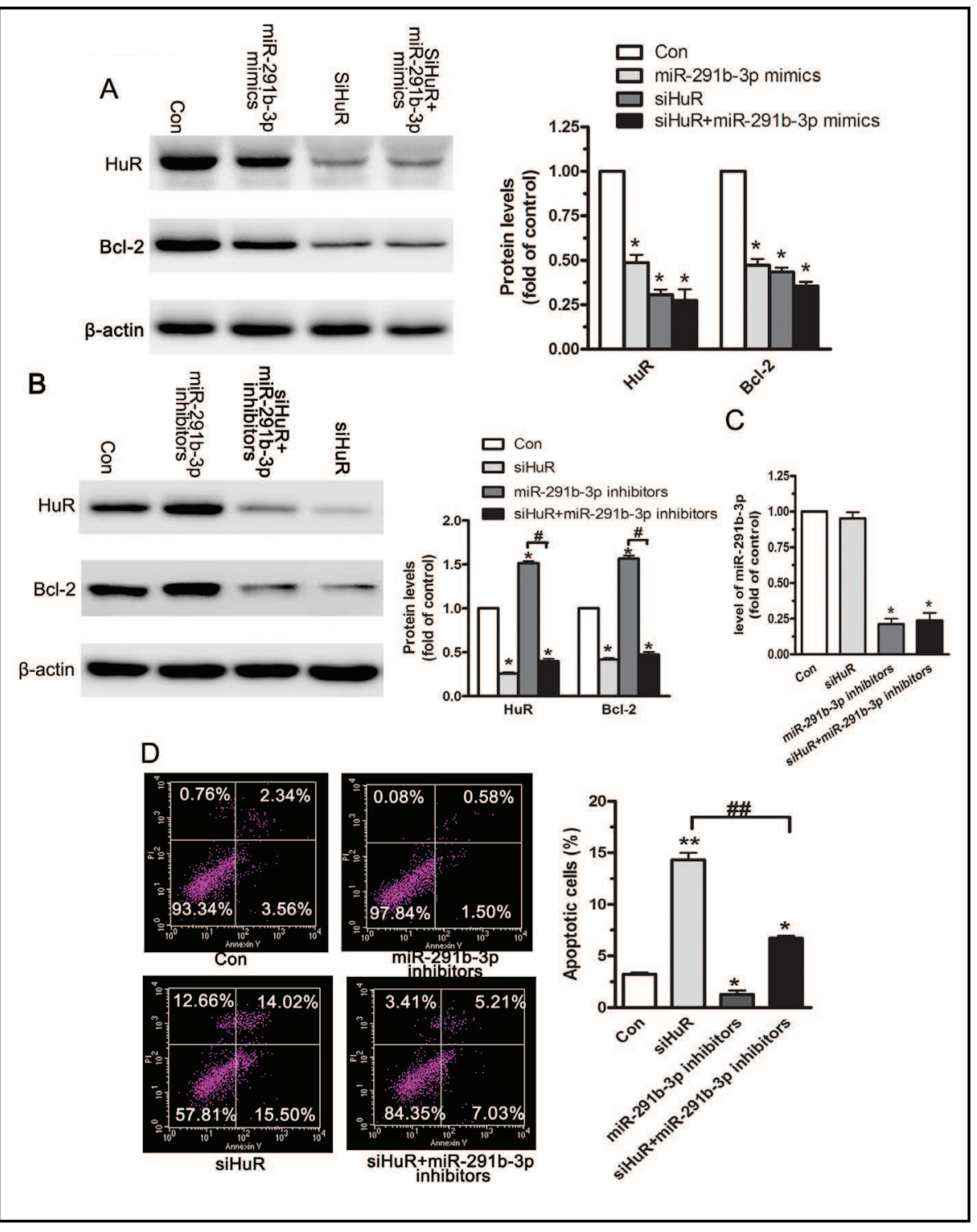

of miR-291b-3p in the NCTC1469 cells, as shown by RT-qPCR (C). Downregulation of miR-291b-3p could induce cell apoptosis when NCTC1469 cells were transfected with siRNA of HuR (D). Data represent the means \pm SEM, $n=3$ independent experiments. ${ }^{*} p<0.05$ versus control. ${ }^{\#} p<0.01$ versus siHuR + miR-291b-3p inhibitors.

(data not shown). These data further confirmed that miR-291b-3p negatively regulates the expression of HuR in NCTC1469 cells.

Suppression of HuR expression induced apoptosis of NCTC1469 cells

Positive regulation of Bcl-2 expression by HuR in various cell types has been previously described [15]. To identify the downstream effects of HuR on cell apoptosis, NCTC1469 cells were transfected with a specific siRNA against HuR. As a result, the HuR expression level was reduced by 75\% compared with the negative control. Additionally, the level of Bcl-2 was also reduced while Bax expression was not changed in these cells (Fig 6. A). Given the results shown in Fig 3. $\mathrm{C}$ and the fact that miRNAs can have multiple target genes, we reasoned that, when the miR-291b-3p mimics were transfected into the NCTC1469 cells, downregulation of Bax was due to the effect of miR-291b-3p on target genes different from HuR. Flow cytometry 


\section{Cellular Physiology and Biochemistry}

Cell Physiol Biochem 2014;33:810-822

\begin{tabular}{l|l}
\hline DOI: $10.1159 / 000358654$ & (C) 2014 S. Karger AG, Basel
\end{tabular}

www.karger.com/cpb

Guo et al.: MiR-291b-3p Induces Cell Apoptosis

analysis of the apoptosis rate after HuR knockdown revealed an $11.29 \%$ increase relative to the control group. These findings indicated that HuR knockdown reduced Bcl-2 expression and enhanced apoptosis in NCTC1469 cells.

MiR-291b-3p induced cell apoptosis by reducing HuR expression

Western blot analysis showed that the levels of $\mathrm{HuR}$ and $\mathrm{Bcl} 2$ were reduced in NCTC1469 cells transfected with either miR-291b-3p mimics or siRNA-HuR (Fig 7. A). In contrast, downregulation of miR-291b-3p elevated the expression of both $\mathrm{HuR}$ and $\mathrm{Bcl} 2$, while downregulation of HuR by siRNA alone reduced Bcl-2 expression. More significantly, downregulation of miR-291b-3p failed to induce Bcl2 expression in NCTC1469 cells that were transfected with siRNA-HuR (Fig 7. B). Moreover, RT-qPCR analysis showed that the downregulation of HuR did not affect the level of miR-291b-3p transcript in NCTC1469 cells. In addition, transfection of the miR-291b-3p inhibitor alone led to reduced apoptosis, while suppression of HuR by siRNA promoted apoptosis, even in cells that were co-transfected with the miR-291b-3p inhibitor (Fig 7. D). Taken together, these results indicated that miR291b-3p promoted NCTC1469 cell apoptosis by reducing HuR expression.

\section{Discussion}

Tumor necrosis factor- $\alpha$ (TNF- $\alpha$ ) is an important proinflammatory cytokine that is linked to various diseases [24]. In hepatocytes, TNF- $\alpha$ binds to TNF receptor 1 (TNFR1) and causes the activation of cellular signaling pathways that control either cell survival or cell death. Although the mechanism by which TNF- $\alpha$ dictates cell fate is not understood, a high concentration of TNF- $\alpha$ in liver causes significant damage to the tissue, as indicated by obvious hepatocyte apoptosis [25]. In the present study, we showed for the first time that the expression of miR-291b-3p is upregulated during TNF- $\alpha$-mediated apoptosis of NCTC1469 cells, suggesting that miR-291b-3p plays an important role in the apoptotic process. We then found that upregulation of miR-291b-3p with specific miRNA mimics resulted in decreased cell viability and increased cell apoptosis, while downregulation of miR-291b-3p with an inhibitor had anti-apoptosis and pro-survival effects.

Evidence of apoptosis regulation by miRNAs has been presented previously. For instance, miR-106a-5p induces astrocytoma cell apoptosis mainly by targeting Fas-activated serine/ threonine kinase (FASTK), and miR-429 enhances cell apoptosis in esophageal carcinoma mainly by suppressing the expression of Bcl-2 and SP-1 [26, 27]. Furthermore, miR-26b has been found to be upregulated in Alzheimer's Disease, and it significantly promotes postmitotic neuronal cell apoptosis [28]. However, the role of miR-291b-3p in apoptosis has never been elucidated. MiR-291b-3p belongs to the murine miR-290 cluster, which includes miR290-3p, miR-291a-3p, miR-291b-3p, miR-292-3p, miR-294 and miR-295 [29]. This cluster has been identified as a $2.2 \mathrm{~kb}$ region on chromosome 7 , and it has been widely reported to regulate different cellular pathways by targeting various target genes [30]. For example, miR-291b-5p and miR-293 suppress embryonic stem cell pluripotency by suppressing the p65 subunit of NF-кB [31].

We sought to elucidate the specific molecular mechanisms behind miR-291b-3pinduced NCTC1469 cell apoptosis. Using four popular miRNA target prediction methods, Miranda, TargetScan, PicTar and RNA22, we failed to identify any apoptosis-related proteins that could be potential targets of miR-291b-3p. However, we found that miR-291b-3p is highly conserved with miR-519b-3p, miR-519c-3p, and miR-519a. It is known that miR519 directly regulates the expression of HuR, which has an mRNA sequence that is highly conserved between Mus musculus and Homo sapiens. HuR is mainly located in the nuclei of resting cells [32]. It has been reported that HuR can bind to ARE-containing mRNAs and be transported to the cytoplasm. The translocation of HuR from nucleus to cytoplasm is an important mechanism for stabilizing target mRNAs [33, 34]. Therefore, we aligned murine miR-291b-3p with the murine HuR mRNA and found that HuR may be a potential target 


\section{Cellular Physiology and Biochemistry}

Cell Physiol Biochem 2014;33:810-822

\begin{tabular}{l|l}
\hline DOI: $10.1159 / 000358654$ & (c) 2014 S. Karger AG, Basel
\end{tabular}

www.karger.com/cpb

Guo et al.: MiR-291b-3p Induces Cell Apoptosis

gene of miR-291b-3p. Direct binding of miR-291b-3p to HuR mRNA was evidenced by the luciferase assay: we found that overexpression of miR-291b-3p significantly impaired the luciferase activity in HEK 293T cells that were transfected with a luciferase reporter vector containing the HuR-CR region. Furthermore, luciferase activity was only slightly reduced in HEK cells that were co-transfected with the luciferase reporter vector containing the HuR3 '-UTR and the miR-291b-3p mimics, suggesting that miR-291b-3p negatively regulates HuR by direct binding to the coding region of HuR mRNA. These results were further validated by western blot, which showed that overexpression of miR-291b-3p reduced the protein level of HuR, while suppression of miR-291b-3p enhanced HuR expression. At present, the regulation of HuR by different miRNAs has been widely explored [35]. For instance, miR-122, miR-548c, miR-494, miR-16, and miR-331 have all been proven to target HuR in various cell types and tumor samples [18, 36-38].

The finding that HuR knockdown promoted NCTC1469 cell apoptosis confirmed the notion that HuR has an anti-apoptotic function. Previous research has already shown that reduced HuR expression confers a growth advantage in various malignant cells [39]. As one of the most important ARE-binding proteins, HuR binds to the ARE sequences in mRNAs with high affinity [40]. However, HuR exerts its effects in two distinctive ways. First, HuR can act as a positive regulator of gene expression by increasing the stability of target mRNAs, such as cox-2, cyclin D1, p21 and so on [13, 41, 42]. However, HuR has also been found to decrease the translation efficiency of some mRNAs, such as TNF $\alpha, c-m y c$, and p27 [43-45]. Recently, $\mathrm{HuR}$ expression level has been suggested to positively correlate with that of Bcl-2 in HeLa cells $[15,46]$. Abdelmohsen et al. indicated that HuR plays a positive role in regulating Bcl-2 mRNA stability and translation efficiency [46]. Similarly, we have now shown that reducing the HuR level contributed to reduced expression of Bcl-2, which then prompted NCTC1469 cell apoptosis by increasing the Bax/Bcl-2 ratio. Interestingly, we also noted higher Bax expression and lower Bcl-2 expression when miR-291b-3p was upregulated. Both proteins belong to the $\mathrm{Bcl}-2$ family, but they have opposite functions during the apoptotic process [47]. Certainly, further investigation is needed to elucidate the mechanisms that lead to Bax upregulation during miR-291b-3p-induced apoptosis. We propose that miR-291b-3p may exert its function on Bax through other target genes than HuR. Nevertheless, our data clearly show that the Bax/Bcl-2 ratio increased when HuR expression was blocked, resulting in cellular apoptosis.

In conclusion, we identified a novel pro-apoptotic role of miR-291b-3p through the modulation of HuR expression and the subsequent effect on Bcl-2 stability in NCTC1469 cells.

\section{Acknowledgements}

This work was supported by grants from National Basic Research Program of China (2012CB517502, 2014CB910503) and National Natural Science Foundation of China (81270887, 81270495).

\section{References}

1 Milenkovic D, Deval C, Gouranton E, Landrier JF, Scalbert A, Morand C, Mazur A: Modulation of miRNA expression by dietary polyphenols in apoE deficient mice: a new mechanism of the action of polyphenols. PLoS One 2012; 7:e29837.

2 Rottiers V, Naar AM: MicroRNAs in metabolism and metabolic disorders. Nat Rev Mol Cell Biol 2012;13:239-250.

- 3 Hu JZ, Huang JH, Zeng L, Wang G, Cao M, Lu HB: Anti-Apoptotic Effect of MicroRNA-21 after Contusion Spinal Cord Injury in Rats. J Neurotrauma 2013;30:1349-1360. 


\section{Cellular Physiology and Biochemistry}

Cell Physiol Biochem 2014;33:810-822

\begin{tabular}{l|l}
\hline DOI: $10.1159 / 000358654$ & (c) 2014 S. Karger AG, Basel
\end{tabular}

Guo et al.: MiR-291b-3p Induces Cell Apoptosis

-4 Farhana L, Dawson MI, Murshed F, Das JK, Rishi AK, Fontana JA: Upregulation of miR-150* and miR-630 induces apoptosis in pancreatic cancer cells by targeting IGF-1R. PLoS One 2013;8:e61015.

-5 Sharma S, Verma S, Vasudevan M, Samanta S, Thakur JK, Kulshreshtha R: The interplay of HuR and miR3134 in regulation of AU rich transcriptome. RNA Biol 2013;10: 1283-1290.

-6 Wang J, Guo Y, Chu H, Guan Y, Bi J, Wang B: Multiple Functions of the RNA-Binding Protein HuR in Cancer Progression, Treatment Responses and Prognosis. Int J Mol Sci 2013;14:10015-10041.

-7 Sahlberg AS, Ruuska M, Granfors K, Penttinen MA: Altered Regulation of ELAVL1/HuR in HLA-B27Expressing U937 Monocytic Cells. PLoS One 2013;8:e70377.

8 Barreau C, Paillard L, Osborne HB: AU-rich elements and associated factors: are there unifying principles? Nucleic Acids Res 2005;33:7138-150.

-9 Peng SS, Chen CY, Xu N, Shyu AB: RNA stabilization by the AU-rich element binding protein, HuR, an ELAV protein. ЕMBO J 1998;17:3461-3470.

10 Rodriguez-Pascual F, Hausding M, Ihrig-Biedert I, Furneaux H, Levy AP, Forstermann U, Kleinert H: Complex contribution of the 3 -untranslated region to the expressional regulation of the human inducible nitric-oxide synthase gene. Involvement of the RNA-binding protein HuR. J Biol Chem 2000;275:2604026049.

11 Chen CY, Xu N, Shyu AB: Highly selective actions of HuR in antagonizing AU-rich element-mediated mRNA destabilization. Mol Cell Biol 2002;22:7268-7278.

-12 Yaman I, Fernandez J, Sarkar B, Schneider RJ, Snider MD, Nagy LE, Hatzoglou M: Nutritional control of mRNA stability is mediated by a conserved AU-rich element that binds the cytoplasmic shuttling protein HuR. J Biol Chem 2002;277:41539-41546.

13 Wang W, Furneaux H, Cheng H, Caldwell MC, Hutter D, Liu Y, Holbrook N, Gorospe M: HuR regulates p21 mRNA stabilization by UV light. Mol Cell Biol 2000;20:760-769.

14 Zou T, Mazan-Mamczarz K, Rao JN, Liu L, Marasa BS, Zhang AH, Xiao L, Pullmann R, Gorospe M, Wang JY: Polyamine depletion increases cytoplasmic levels of RNA-binding protein HuR leading to stabilization of nucleophosmin and p53 mRNAs. J Biol Chem 2006;281:19387-19394.

15 Ishimaru D, Ramalingam S, Sengupta TK, Bandyopadhyay S, Dellis S, Tholanikunnel BG, Fernandes DJ, Spicer EK: Regulation of Bcl-2 expression by HuR in HL60 leukemia cells and A431 carcinoma cells. Mol Cancer Res 2009; 7:1354-1366.

-16 Leucci E, Zriwil A, Gregersen LH, Jensen KT, Obad S, Bellan C, Leoncini L, Kauppinen S, Lund AH: Inhibition of miR-9 de-represses HuR and DICER1 and impairs Hodgkin lymphoma tumour outgrowth in vivo. Oncogene 2012;31:5081-5089.

17 Kojima K, Fujita Y, Nozawa Y, Deguchi T, Ito M: MiR-34a attenuates paclitaxel-resistance of hormonerefractory prostate cancer PC3 cells through direct and indirect mechanisms. Prostate 2010;70:15011512.

18 Xu F, Zhang X, Lei Y, Liu X, Liu Z, Tong T, Wang W: Loss of repression of HuR translation by miR-16 may be responsible for the elevation of HuR in human breast carcinoma. J Cell Biochem 2010;111:727-734.

19 Al-Ahmadi W, Al-Ghamdi M, Al-Souhibani N, Khabar KS: miR-29a inhibition normalizes HuR overexpression and aberrant AU-rich mRNA stability in invasive cancer. J Pathol 2013;230:28-38.

20 Prislei S, Martinelli E, Mariani M, Raspaglio G, Sieber S, Ferrandina G, Shahabi S, Scambia G, Ferlini C: MiR200c and HuR in ovarian cancer. BMC Cancer 2013;13:72.

-21 Abdelmohsen K, Srikantan S, Kuwano Y, Gorospe M: miR-519 reduces cell proliferation by lowering RNAbinding protein HuR levels. Proc Natl Acad Sci 2008;105:20297-20302.

-22 Dou L, Zhao T, Wang L, Huang X, Jiao J, Gao D, Zhang H, Shen T, Man Y, Wang S, Li J: miR-200s contribute to interleukin-6 (IL-6)-induced insulin resistance in hepatocytes. J Biol Chem 2013;288:22596-22606.

-23 Abdelmohsen K, Kim MM, Srikantan S, Mercken EM, Brennan SE, Wilson GM, Cabo R, Gorospe M: miR-519 suppresses tumor growth by reducing HuR levels. Cell Cycle 2010;9:1354-1359.

24 Minero VG, Khadjavi A, Costelli P, Baccino FM, Bonelli G: JNK activation is required for TNFalpha-induced apoptosis in human hepatocarcinoma cells. Int Immunopharmacol 2013;17:92-98.

25 Shuh M, Bohorquez H, Loss GE, Jr., Cohen AJ: Tumor Necrosis Factor-alpha: Life and Death of Hepatocytes During Liver Ischemia/Reperfusion Injury. Ochsner J 2013;13:119-130.

26 Wang Y, Li M, Zang W, Ma Y, Wang N, Li P, Wang T, Zhao G: MiR-429 up-regulation induces apoptosis and suppresses invasion by targeting Bcl-2 and SP-1 in esophageal carcinoma. Cell Oncol 2013;36:385-394. 


\section{Cellular Physiology and Biochemistry}

Cell Physiol Biochem 2014;33:810-822

\begin{tabular}{l|l}
\hline DOI: $10.1159 / 000358654$ & (c) 2014 S. Karger AG, Basel
\end{tabular}

Guo et al.: MiR-291b-3p Induces Cell Apoptosis

27 Zhi F, Zhou G, Shao N, Xia X, Shi Y, Wang Q Zhang Y, Wang R, Xue L, Wang S, Wu S, Peng Y, Yang Y: miR-106a$5 p$ Inhibits the Proliferation and Migration of Astrocytoma Cells and Promotes Apoptosis by Targeting FASTK. PLoS One 2013;8:e72390.

-28 Absalon S, Kochanek DM, Raghavan V, Krichevsky AM: MiR-26b, Upregulated in Alzheimer's Disease, Activates Cell Cycle Entry, Tau-Phosphorylation, and Apoptosis in Postmitotic Neurons. J Neurosci 2013;33:14645-14659.

-29 Marson A, Levine SS, Cole MF, Frampton GM, Brambrink T, Johnstone S, Guenther MG, Johnston WK, Wernig M, Newman J, Calabrese JM, Dennis LM, Volkert TL, Gupta S, Love J, Hannett N, Sharp PA, Bartel DP, Jaenisch $\mathrm{R}$, Young RA: Connecting microRNA genes to the core transcriptional regulatory circuitry of embryonic stem cells. Cell 2008;134:521-533.

-30 Zheng GX, Ravi A, Calabrese JM, Medeiros LA, Kirak O, Dennis LM, Jaenisch R, Burge CB, Sharp PA: A latent pro-survival function for the mir-290-295 cluster in mouse embryonic stem cells. PLoS Genet 2011; 7:e1002054.

-31 Luningschror P, Stocker B, Kaltschmidt B, Kaltschmidt C: miR-290 cluster modulates pluripotency by repressing canonical NF-kappaB signaling. Stem Cells 2012;30:655-664.

-32 Yi X, Zhou Y, Zheng W, Chambers SK: HuR expression in the nucleus correlates with high histological grade and poor disease-free survival in ovarian cancer. Aust N Z J Obstet Gynaecol 2009;49:93-98.

-33 Zhu Z, Wang B, Bi J, Zhang C, Guo Y, Chu H, Liang X, Zhong C, Wang J: Cytoplasmic HuR expression correlates with P-gp, HER-2 positivity, and poor outcome in breast cancer. Tumour Biol 2013;34:2299-2308.

-34 Hostetter C, Licata LA, Witkiewicz A, Costantino CL, Yeo CJ, Brody JR, Keen JC: Cytoplasmic accumulation of the RNA binding protein HuR is central to tamoxifen resistance in estrogen receptor positive breast cancer cells. Cancer Biol Ther 2008;7:1496-1506.

-35 Palanisamy V, Jakymiw A, Van Tubergen EA, D'Silva NJ, Kirkwood KL: Control of cytokine mRNA expression by RNA-binding proteins and microRNAs. J Dent Res 2012;91:651-658.

-36 Bhattacharyya SN, Habermacher R, Martine U, Closs EI, Filipowicz W: Relief of microRNA-mediated translational repression in human cells subjected to stress. Cell 2006;125:1111-1124.

- 37 Tominaga K, Srikantan S, Lee EK, Subaran SS, Martindale JL, Abdelmohsen K, Gorospe M: Competitive regulation of nucleolin expression by HuR and miR-494. Mol Cell Biol 2011;31:4219-4231.

- 38 Epis MR, Barker A, Giles KM, Beveridge DJ, Leedman PJ: The RNA-binding protein HuR opposes the repression of ERBB-2 gene expression by microRNA miR-331-3p in prostate cancer cells. J Biol Chem 2011;286:41442-41454.

39 Dalmau J, Furneaux HM, Cordon-Cardo C, Posner JB: The expression of the Hu (paraneoplastic encephalomyelitis/sensory neuronopathy) antigen in human normal and tumor tissues. Am J Pathol 1992;141:881-886.

-40 Al-Ahmadi W, Al-Ghamdi M, Al-Haj L, Al-Saif M, Khabar KS: Alternative polyadenylation variants of the RNA binding protein, HuR: abundance, role of AU-rich elements and auto-Regulation. Nucleic Acids Res 2009;37:3612-3624.

-41 Kim GY, Lim SJ, Kim YW: Expression of HuR, COX-2, and survivin in lung cancers; cytoplasmic HuR stabilizes cyclooxygenase-2 in squamous cell carcinomas. Mod Pathol 2011;24:1336-1347.

-42 Yuan Z, Sanders AJ, Ye L, Wang Y, Jiang WG: Knockdown of human antigen R reduces the growth and invasion of breast cancer cells in vitro and affects expression of cyclin D1 and MMP-9. Oncol Rep 2011;26:237-245.

-43 Nabors LB, Suswam E, Huang Y, Yang X, Johnson MJ, King PH: Tumor necrosis factor alpha induces angiogenic factor up-regulation in malignant glioma cells: a role for RNA stabilization and HuR. Cancer Res 2003;63:4181-4187.

-44 Talwar S, Jin J, Carroll B, Liu A, Gillespie MB, Palanisamy V: Caspase-mediated cleavage of RNA-binding protein HuR regulates c-Myc protein expression after hypoxic stress. J Biol Chem 2011;286:32333-32343.

-45 Kullmann M, Gopfert U, Siewe B, Hengst L: ELAV/Hu proteins inhibit p27 translation via an IRES element in the p27 5'UTR. Genes Dev 2002;16:3087-3099.

46 Abdelmohsen K, Lal A, Kim HH, Gorospe M: Posttranscriptional orchestration of an anti-apoptotic program by HuR. Cell Cycle 2007;6:1288-1292.

-47 Hardwick JM, Soane L: Multiple functions of BCL-2 family proteins. Cold Spring Harb Perspect Biol 2013;5:pii: a008722. doi: 10.1101/cshperspect.a008722. 